
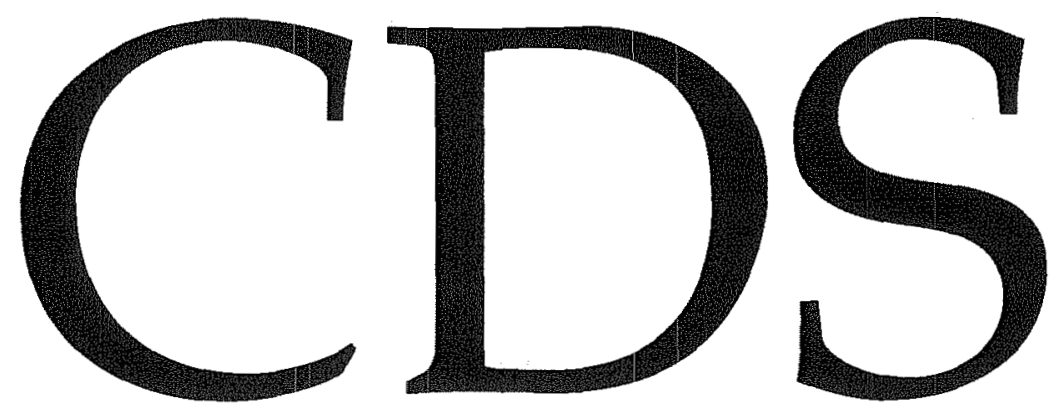

TECHNICAL MEMORANDUM NO. CIT-CDS 93-019

October 15, 1993

"Interconnection of Uncertain Behavioral Systems for Robust Control"

Raffaello D'Andrea and Fernando Paganini

Control and Dynamical Systems California Institute of Technology

Pasadena, CA 91125 


\title{
Interconnection of Uncertain Behavioral Systems for Robust Control
}

\author{
Raffaello D'Andrea Fernando Paganini \\ Electrical Engineering, M/S 116-81 \\ California Institute of Technology \\ Pasadena, CA 91125
}

October 15,1993

\begin{abstract}
This paper attempts to relate robust control and behavioral frameworks by incorporating structured uncertainty into the description of behavioral systems. Behavioral equations are expressed as linear fractional transformations (LFTs) on an uncertainty structure, and a method of interconnection is outlined. A method for obtaining inputoutput maps from LFT representations of behavioral systems is also presented. This extension of the behavioral framework is compatible with existing robust control methods, such as $\mu$ analysis, which can be used to provide robustness tests in behaviors. A simple example is presented that illustrates some of the issues which arise in this extension.
\end{abstract}

\section{Introduction}

A major theme in robust control has been to supply the engineer with a theoretical and computational framework that handles a rich variety of modeling uncertainty so that physically motivated uncertainty descriptions can be treated in a natural manner. In particular, it has been important to provide computational tools that analyze systems with mixtures of unstructured uncertainties and possibly large numbers of uncertain real parameters. Behavioral models are in turn very natural when modeling physical systems from first principles, or when a large system is built up from subsystem models. While the final interconnected model used in a robust control design may have well-defined inputs and outputs, it is almost always the case that components are modeled in terms of mass, momentum, or energy balances or physical laws such as Newton's second law, Ohm's law, and so on. These components do not have a pre-specified signal direction, and forcing them to be input-output operators assumes a knowledge of the ultimate function of the device. The reader is referred to [1] for a detailed exposition of the issues outlined above.

The behavioral setting provides a convenient framework for connecting modeling and robust control. Uncertainty can be incorporated in the description of behavioral systems 
via linear fractional transformations (LFTs). By describing these systems as LFTs, existing robust control methods, such as $\mu$ analysis and synthesis, can be used to provide robustness tests in behaviors.

\section{Background}

\subsection{Behavioral Systems}

The behavioral framework has been extensively described and developed by Willems in [2], of which a summary relevant to this paper may be found in [1]. In the interest of completeness, a brief summary follows. A dynamical system is viewed as a family of trajectories, and is defined as a triple $\Sigma=(\mathbb{T}, \mathbb{W}, \mathcal{B})$, with $T$ a subset of $\mathbb{R}$ called the time axis, $\mathbb{W}$ the signal space, and $\mathcal{B}$ a subset of $\mathbb{W}^{\mathbb{T}}$ called the behavior.

In the absence of uncertainty, we will be concerned with continuous-time, linear, time invariant, finite dimensional dynamical systems. In particular, we require that $T=\mathbb{R}^{+}$and $\mathbb{W}=\mathbb{R}^{q}$. In [3], it is shown that this is equivalent to requiring $\mathcal{B}$ to be the kernel of a polynomial operator in the differentiation operator, $\frac{d}{d t}$. We will also restrict ourselves to time trajectories $w$ that are locally square integrable:

$$
\int_{\Omega}\|w\|^{2} d t<\infty
$$

for all compact sets $\Omega \subset \mathbb{R}^{+}$, which will be denoted $w \in L_{2}^{\text {loc }}$. We will refer to the class of dynamical systems with the above properties as $\mathcal{L}$.

Although continuous time systems are considered in this paper, most of the results presented can either be applied directly to discrete time systems, or can be trivially extended to the discrete time case. The same can be said for the signal space chosen. We have chosen to consider time trajectories that are locally square integrable, but the results generalize to all $L_{p}^{l o c}$ spaces.

\subsection{Linear Fractional Transformations}

LFTs are a class of general linear feedback loops, and can be pictorially depicted as follows:

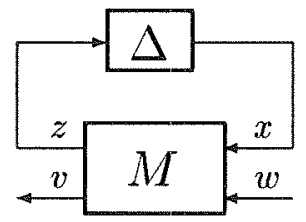

Figure 1: Linear Fractional Transformation

We will be interested in cases where $M$ is a constant, finite dimensional matrix and $\Delta$ is, as of yet, an unspecified operator. If we partition $M$ as 
we have the following loop equations:

$$
M=\left[\begin{array}{ll}
A & B \\
C & D
\end{array}\right]
$$

$$
\begin{aligned}
& z=A x+B w \\
& v=C x+D w \\
& x=\Delta z
\end{aligned}
$$

If the inverse of $(I-A \Delta)$ exists, the vectors $v$ and $w$ satisfy $v=\mathcal{S}(\Delta, M) w$, where

$$
\mathcal{S}(\Delta, M)=D+C \Delta(I-A \Delta)^{-1} B
$$

$\mathcal{S}(\Delta, M)$ is called an LFT between $\Delta$ and $M$. $\Delta$ will usually belong to an allowable set of operators, $\Delta$. We will consider $\Delta$ structures of the form

$$
\Delta=\left\{\operatorname{diag}\left[\int I, \Delta_{\mathbf{u}}\right]\right\}
$$

where $\Delta_{\mathbf{u}}$ is a structured set of bounded, linear operators on $L_{2}^{\text {loc }}$, referred to as the uncertainty block. Often we will need norm bounded subsets of $\Delta_{u}$, and we introduce the notation

$$
\mathbf{B}_{\Delta, \epsilon}=\left\{\Delta \in \Delta:\left\|\Delta_{u}\right\|<\epsilon\right\}
$$

Note the norm bound is only on the uncertainty portion of $\Delta, \Delta_{\mathbf{u}}$.

Many systems with uncertainty can be represented using LFTs. For example, uncertainty in the physical quantities of a system, such as mass or capacitance, can easily be written as LFTs on an uncertainty block. The same is true for arbitrary operators on variables, which might arise, for example, when modeling the effects of friction on a system. The interested reader is referred to [1] and [5] for examples of the type of uncertainty that may be represented by LFTs.

\section{Representation}

In order to include uncertainty into our system description, it is desirable to first explore how systems without uncertainty may be modeled. Incorporating uncertainty into our system description should then ideally be a natural extension of the existing framework.

Consider the following equations:

$$
\begin{aligned}
& \dot{x}=A x+B w \\
& 0=C x+D w
\end{aligned}
$$

where $A, B, C, D$ are constant, finite dimensional matrices. Then it can be shown (see [4]) that the behavior of a dynamical system $\Sigma$ in $\mathcal{L}$ can be characterized as

$$
\mathcal{B}=\left\{w \in L_{2}^{\text {loc }} \mid \exists x \in L_{2}^{\text {loc }} \text { for which (7) is satisfied }\right\}
$$

for some $A, B, C$, and $D$. Equations (7) are referred to as an output nulling (ON) representation of $\Sigma$, and is denoted $\{A, B, C, D\}$. There are many other equivalent state space representations of dynamical systems in $\mathcal{L}$, see [6] and [7] for example. As will be seen, however, $\mathrm{ON}$ representations can be naturally extended to incorporate uncertainty into our system description.

The following definitions apply to the set $\{A, B, C, D\}$ : 
Definition 1 ON Representation $\{A, B, C, D\}$ of $\Sigma$ is said to be

Dependent if, $\forall s \in \mathbb{C}, D+C(s I-A)^{-1} B$ is not surjective

Regular if $D$ is surjective

Singular if not dependent and not regular

It follows that regular representations can never be dependent, and vice-versa. In the singular case, the rank of $D+C(s I-A)^{-1} B$ is normally full but drops at $s=\infty$. It should be stressed that the above are properties of the ON representation of $\Sigma$, not of $\Sigma$ itself. As will be seen, it is desirable to work with regular representations of systems in $\mathcal{L}$. Given an ODE, it is a straightforward matter to obtain from it a regular $O N$ representation. Consider the following ODE

$$
p_{1}(\sigma) w_{1}+p_{2}(\sigma) w_{2}+\cdots p_{q}(\sigma) w_{q}=0
$$

where $p_{j}$ is a polynomial in $\sigma=\frac{d}{d t}$, and $w_{j}$ are the manifest variables. Let $l$ be the highest power of $\sigma$ appearing in equation (9). Then define $\overline{p_{j}}\left(\sigma^{-1}\right)=p_{j}(\sigma) \sigma^{-1}$. It follows that polynomials $\overline{p_{j}}$ can be expressed as

$$
\overline{p_{j}}=\sum_{i=1}^{l+1} b_{i j} \sigma^{i-l-1}, b_{l+1, j} \neq 0 \text { for some } j
$$

Now define

$$
\begin{aligned}
& A=\left[\begin{array}{ccccc}
0 & 0 & 0 & \cdots & 0 \\
1 & 0 & 0 & \cdots & 0 \\
0 & 1 & 0 & \cdots & 0 \\
& & \vdots & & \\
0 & 0 & \cdots & 1 & 0
\end{array}\right] \in \mathbb{R}^{l \times l} \\
& B=\left[b_{i j}\right] \in \mathbb{R}^{l \times q}, i=1,2, \cdots, l \\
& C=\left[\begin{array}{lll}
0 & 0 & \cdots
\end{array}\right], \in \mathbb{R}^{1 \times l} \\
& D=\left[b_{l+1, j}\right] \in \mathbb{R}^{1 \times q}
\end{aligned}
$$

Then the set of all solutions $w$ of

$$
\begin{aligned}
& \dot{x}=A x+B w \\
& 0=C x+D w
\end{aligned}
$$

is equivalent to the set of all solutions of equation (9). Furthermore, $\{A, B, C, D\}$ is regular, by construction. It is also minimal in the sense that matrices $A, B, C, D$ cannot be made smaller, since $(C, A)$ is observable and $D$ is surjective, a result proved in [4].

When more than one ODE constitutes a system, a regular ON representation may be obtained by first using the above procedure for each ODE, and then using the interconnection procedure outlined in the interconnection section of this paper.

An output nulling representation may be interpreted as an LFT between a constant matrix and an integration operator: 


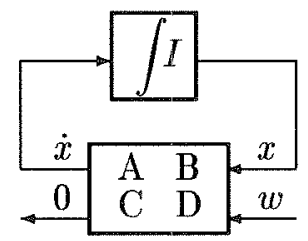

Figure 2: ON Representation as LFT

where it is understood that $x=\int_{0}^{t} \dot{x} d \tau+x(0)$, ie., the initial values of the integrator states need not be 0 .

The above LFT interpretation motivates how uncertainty should be incorporated into our description, and leads to the following definition:

Definition 2 An uncertain dynamical system is in general a parameterized family $\Sigma_{\Delta}=$ $\left\{\Sigma_{\Delta}: \Delta \in \Delta\right\}$ of dynamical systems, and is denoted by a quadruple $\Sigma_{\Delta}=\left(\mathbb{T}, \mathbb{W}, \Delta, \mathcal{B}_{\Delta}\right)$. The parameter $\Delta \in \Delta$ incorporates the system uncertainty.

We will consider uncertain dynamical systems for which the parameterized behavior may be expressed as

$$
\mathcal{B}_{\Delta}=\left\{w \in L_{2}^{\text {loc }} \mid \mathcal{S}(\Delta, M) w=0\right\}
$$

where $M$ is a constant matrix, referred to as the representation matrix, and $\Delta$ varies in a set of structured operators, $\Delta$. This representation is denoted $(\Delta, M)$, and is called a Generalized Output Nulling (GON) representation of $\Sigma_{\Delta}$.

Setting $\Delta=\int I$ yields the same behavior as ON representation $\{A, B, C, D\}$, where the representation matrix $M$ is partitioned accordingly. In the presence of uncertainty, $\Delta$ is of the form

$$
\Delta=\left\{\operatorname{diag}\left[\int I, \Delta_{\mathbf{u}}\right]\right\}
$$

This choice of $\Delta$ induces a natural partition of representation matrix $M$ :

$$
M=\left[\begin{array}{ccc}
A_{n} & A_{n u} & B_{n} \\
A_{u n} & A_{u} & B_{u} \\
C_{n} & C_{u} & D
\end{array}\right]
$$

where subscript $n$ implies nominal and subscript $u$ implies uncertain.

As in the case of no uncertainty, a GON representation is said to be regular if $D$ is surjective. We will be interested in uncertain dynamical systems for which there exists a regular GON representation. There exist systems for which the regularity assumption is not valid. Consider the following equation:

$$
\delta x=0
$$

where $\delta$ is a real parameter, and $x$ a variable. It can be easily verified that there does not exist a GON representation of the above with $D$ non-zero. In a sense, the above is a result of choosing a "bad" value for the nominal coefficient multiplying $x$, in this case 0 . The 
assumption of regularity implies that the system behavior at $\Delta_{u}=0$ is in some sense similar to the system behavior in a neighborhood of $\Delta_{u}=0$. In the simple example above, this problem could be remedied by defining a new real parameter $\bar{\delta}=\delta-1$, and rewriting the above equation as $(1+\bar{\delta}) x=0$. It should be noted, however, that the nominal constraint is now $x=0$, as opposed to no constraint on $x$ when $\delta=0$.

There are some transformations on the representation matrix which are behavior preserving. We have the following result:

Lemma 1 Given representation matrix $M=\left[\begin{array}{cc}A & B \\ C & D\end{array}\right], \bar{M}$ is an equivalent representation matrix if

$$
\bar{M}=\left[\begin{array}{cc}
T^{-1}(A+L C) T & T^{-1}(B+L D) \\
P C T & P D
\end{array}\right]
$$

where $L$ is any matrix, $P$ is any square, invertible matrix, and $T$ is any square, invertible matrix such that $T^{-1} \Delta T=\Delta \forall \Delta \in \Delta$.

The proof of the above is a direct extension of Theorem 3.20 in [4]. Matrices $L, P$, and $T$ can be interpreted as follows: $L$ injects the output of the LFT into the state equation. Since the output is required to be zero, the behavior is obviously preserved. Similarly, bijectively mapping the output of the LFT by $P$ to another output space does not alter the behavior, since the output is zero. Finally, $T$ can be interpreted as a state transformation.

In the absence of uncertainty, and when the representation is minimal in the sense that matrix $M$ is as small as possible, the implication in the above lemma goes the other way also; all equivalent representations are related as in equation (17). Whether this is also true for minimal representations of uncertain systems is an open research question, aspects of which are considered in [8].

\section{Interconnection}

Interconnection of systems without uncertainty in a behavioral setting is formally defined in [2]. For the purpose of this paper, we will use the following simplified definition:

Definition 3 The interconnection of two systems with the same signal space $\Sigma_{1}=\left(\mathbb{R}^{+}, \mathbb{R}^{q}, \mathcal{B}_{1}\right)$ and $\Sigma_{2}=\left(\mathbb{R}^{+}, \mathbb{R}^{q}, \mathcal{B}_{2}\right)$ is denoted $\Sigma_{1} \wedge \Sigma_{2}$, where $\Sigma_{1} \wedge \Sigma_{2}=\left(\mathbb{R}^{+}, \mathbb{R}^{q}, \mathcal{B}_{1} \cap \mathcal{B}_{2}\right)$

Thus interconnection can be interpreted as the intersection of behaviors, or as combining constraint equations. The requirement that the signal spaces of two systems be equal before interconnection can easily be accommodated by augmenting the signal space of each system to include the other system's variables.

The machinery of interconnection presented below may be used when building a model from first principles, or when systems are connected together. Mathematically they are equivalent, since both consist of combining constraint equations. As stated in the previous section, a system of ODEs can be represented by a regular ON representation by simply building ON representations of each ODE, and then interconnecting them. 
Proposition 1 Given regular $O N$ representations $\left\{A_{1}, B_{1}, C_{1}, D_{1}\right\}$ and $\left\{A_{2}, B_{2}, C_{2}, D_{2}\right\}$ of two systems $\Sigma_{1}$ and $\Sigma_{2}$ with the same signal space, an $O N$ representation of their interconnection $\Sigma_{1} \wedge \Sigma_{2}$ is

$$
S=\left\{\left[\begin{array}{cc}
A_{1} & 0 \\
0 & A_{2}
\end{array}\right],\left[\begin{array}{l}
B_{1} \\
B_{2}
\end{array}\right],\left[\begin{array}{cc}
C_{1} & 0 \\
0 & C_{2}
\end{array}\right],\left[\begin{array}{c}
D_{1} \\
D_{2}
\end{array}\right]\right\}
$$

The above follows immediately from the definition of interconnection, and can be trivially extended to the interconnection of more than two systems.

There are three distinct cases which may arise. $S$ may be dependent, singular, or regular. A dependent representation may be obtained when there are redundant constraint equations. In electrical circuits, this can easily occur when there are too many loop or node equations. It is desirable in these cases to simply omit the redundant equations. Singular interconnections arise when the interconnection forces an algebraic constraint between states in the subsystems.

We can think of interconnection in two contexts. First, when interconnection is simply an artifice of our modeling process, where we have broken the system into subsystems. In this case, a singular interconnection is simply a flag that our states are constrained and therefore we might want to simplify the model. A simple example is the modeling of two capacitors in parallel using two states for the voltages across the capacitors. There exists an algebraic constraint on the states that requires them to be equal for all time.

Second, when a physical interconnection is established at a particular instant in time. In this case, a singular interconnection would require that the states be matched in advance, otherwise we will have a transient phenomenon which is not modeled and is potentially damaging. If two capacitors are connected together in parallel, one must be aware that unless the residual charges on the two capacitors are compatible, a large current will flow at the time of contact. We will not deal here with describing such phenomena, which are considered in [9].

We can extend Definition 3 to include uncertainty:

Definition 4 The interconnection of two systems with the same signal space $\Sigma_{\Delta_{1}}=\left(\mathbb{R}^{+}, \mathbb{R}^{q}, \Delta_{1}, \mathcal{B}_{\Delta_{1}}\right)$ and $\Sigma_{\Delta_{2}}=\left(\mathbb{R}^{+}, \mathbb{R}^{q}, \Delta_{\mathbf{2}}, \mathcal{B}_{\Delta_{2}}\right)$ is denoted $\Sigma_{\Delta_{1}} \wedge \Sigma_{\Delta_{2}}$, where $\Sigma_{\Delta_{1}} \wedge \Sigma_{\Delta_{2}}=\left(\mathbb{R}^{+}, \mathbb{R}^{q},\left(\Delta_{\mathbf{1}}, \Delta_{\mathbf{2}}\right), \mathcal{B}_{\Delta_{1}} \cap\right.$ $\left.\mathcal{B}_{\Delta_{2}}\right)$.

A similar extension of Proposition 1 yields

Proposition 2 Given regular GON representations $\left(\Delta_{1}, M_{1}\right)$ and $\left(\Delta_{2}, M_{2}\right)$ of two systems $\Sigma_{\Delta_{1}}$ and $\Sigma_{\Delta_{2}}$ with the same signal space, a GON representation of their interconnection $\Sigma_{\Delta 1} \wedge \Sigma_{\Delta_{2}}$ is

$$
\begin{aligned}
M & =\left[\begin{array}{ccccc}
A_{1_{n}} & 0 & A_{1_{n u}} & 0 & B_{1_{n}} \\
0 & A_{2_{n}} & 0 & A_{2_{n u}} & B_{2_{n}} \\
A_{1_{u n}} & 0 & A_{1_{u}} & 0 & B_{1_{u}} \\
0 & A_{2_{u n}} & 0 & A_{2_{u}} & B_{2_{u}} \\
C_{1_{n}} & 0 & C_{1_{u}} & 0 & D_{1} \\
0 & C_{2_{n}} & 0 & C_{2_{u}} & D_{2}
\end{array}\right] \\
\Delta & =\left\{\operatorname{diag}\left[\int I, \Delta_{\mathbf{u}_{1}}, \Delta_{\mathbf{u}_{\mathbf{u}}}\right]\right\}
\end{aligned}
$$


where

$$
\begin{aligned}
M_{i} & =\left[\begin{array}{ccc}
A_{i_{n}} & A_{i_{n u}} & B_{i_{n}} \\
A_{i_{u n}} & A_{i_{u}} & B_{i_{u}} \\
C_{i_{n}} & C_{i_{u}} & D_{i}
\end{array}\right] \\
\Delta_{\mathbf{i}} & =\left\{\operatorname{diag}\left[\int I, \Delta_{\mathbf{u}_{\mathbf{i}}}\right]\right\}
\end{aligned}
$$

In order to check whether the interconnection of two systems is regular or not, we must start out with regular representations. After interconnection, we would also like to make the resulting representation regular. What follows is one step of a recursive procedure which enables us to construct a regular GON representation from one that is not.

Consider a GON representation $(\Delta, \hat{M})$ of $\Sigma_{\Delta}$, where $\hat{M}$ is partitioned as in (15). We then have the following equations:

$$
\begin{aligned}
\dot{\hat{x}} & =\hat{A}_{n} \hat{x}+\hat{A}_{n u} \Delta_{u} z+\hat{B}_{n} w \\
z & =\hat{A}_{u n} \hat{x}+\hat{A}_{u} \Delta_{u} z+\hat{B}_{u} w \\
0 & =\hat{C}_{n} \hat{x}+\hat{C}_{u} \Delta_{u} z+\hat{D} w
\end{aligned}
$$

If $\hat{D}$ is not surjective, there exists a non-singular matrix $P$ such that the last row of $P \hat{D}$ is zero. Furthermore, since $P$ is non-singular, the solutions to the above equations are not changed if the last equation of (20) is pre-multiplied by $P$. Therefore it can be assumed, without loss of generality, that the last row of $\hat{D}$ is zero, which results in the equation

$$
\hat{C}_{n_{r}} \hat{x}+\hat{C}_{u_{r}} \Delta_{u} z=0
$$

where $\hat{C}_{n_{r}}$ is the last row of matrix $\hat{C}_{n}$, and $\hat{C}_{u_{r}}$ is the last row of matrix $\hat{C}_{u}$.

If $\hat{C}_{n_{r}}$ and $\hat{C}_{u_{r}}$ are zero, the GON representation is said to be dependent, which generalizes our previous definition of dependent. In the case of a dependent representation, another representation exists with at least one fewer constraint equations. One such representation can obtained by truncating the last rows of $\hat{C}_{n}, \hat{C}_{u}$, and $\hat{D}$. If the truncated $\hat{D}$ is still not surjective, the process may be reiterated.

When $\hat{C}_{u_{r}}$ is zero and $\hat{C}_{n_{r}}$ is not equal to zero, there exists an invertible matrix $T$ such that $\hat{C}_{n_{r}} T=\left[\begin{array}{llll}1 & 0 & \cdots & 0\end{array}\right]$. Letting $x=T^{-1} \hat{x}$ results in the following equivalent representation matrix

$$
\left[\begin{array}{ccc}
A_{n} & A_{n u} & B_{n} \\
A_{u n} & A_{u} & B_{u} \\
C_{n} & C_{u} & D
\end{array}\right]=\left[\begin{array}{ccc}
T^{-1} \hat{A_{n}} T & T^{-1} \hat{A}_{n u} & T^{-1} \hat{B}_{n} \\
\hat{A}_{u n} T & \hat{A}_{u} & \hat{B}_{u} \\
\hat{C}_{n} T & \hat{C}_{u} & \hat{D}
\end{array}\right]
$$

We then have the following:

$$
\begin{aligned}
\dot{x} & =A_{n} x+A_{n u} \Delta_{u} z+B_{n} w \\
z & =A_{u n} x+A_{u} \Delta_{u} z+B_{u} w \\
0 & =C_{n_{i}} x+C_{u_{i}} \Delta_{u} z+D_{i} w \quad i=1,2, \cdots, r-1 \\
0 & =x_{1}
\end{aligned}
$$

Differentiating the last equation yields

$$
0=A_{n_{1}} x+A_{n u_{1}} \Delta_{u} z+B_{n_{1}} w
$$


where $A_{n_{1}}$ is the first row of $A_{n}, A_{n u_{1}}$ is the first row of $A_{n u}$, and $B_{n_{1}}$ is the first row of $B_{n}$. Note that the above equation implies that $x_{1}=$ constant, not 0 , therefore $x_{1}=0$ must be incorporated into our state space equations. Therefore representation

$$
M=\left[\begin{array}{cc}
m_{11} & M_{12} \\
M_{21} & \bar{M}
\end{array}\right]=\left[\begin{array}{ccc}
A_{n} & A_{n u} & B_{n} \\
A_{u n} & A_{u} & B_{u} \\
C_{n_{1}} & C_{u_{1}} & D_{1} \\
& \vdots & \\
C_{n_{r-1}} & C_{u_{r-1}} & D_{r-1} \\
A_{n_{1}} & A_{n u_{1}} & B_{n_{1}}
\end{array}\right]
$$

along with the constraint $x_{1}=0$ yields the same set of solutions $w$ as $\hat{M}$. But the constraint $x_{1}=0$ can be imposed directly on representation matrix $M$ by removing the first row and first column of $M$, resulting in representation matrix $\bar{M}$ which has one less integrator state.

It immediately follows that the above step could be repeated until a regular GON representation is obtained:

Proposition 3 Assuming that $\hat{C}_{u_{r}}=0$ whenever $\hat{D}_{r}=0$ in the above step of the recursive reduction procedure, a regular GON representation can be obtained from a non-regular $G O N$ representation $\hat{M}$ in at most $N$ steps, where $N$ is the dimension of matrix $\hat{A}_{n}$

If $\hat{C}_{u_{r}}$ is not equal to zero when $\hat{D}_{r}$ is zero, the reduction procedure as outlined will not work, even though a regular representation may exist. All the examples which we have devised that cause the reduction procedure to fail with a constant $P$ matrix, however, have had no physical interpretation.

The shortcoming of the procedure is that multiplying the last row of (20) by an invertible, constant, matrix $P$ is not general enough. In fact, $P$ can be any invertible operator, not necessarily constant. For the case where the uncertainty structure consists exclusively of scalar times identity blocks, which arise naturally when dealing with parametric uncertainty, we have devised a method which enables us to continue the reduction procedure when a regular representation exists, at the cost of greatly increasing the complexity of the uncertainty structure. The final representation is also only valid locally about $\Delta_{u}=0$. The main idea is to close the LFT loop on the uncertainty structure, and reduce $\hat{D}\left(\Delta_{u}\right)$ to row reduced echelon form by left multiplying with matrix $P\left(\bar{\Delta}_{u}\right)$, where $\bar{\Delta}_{u}$ is an augmented version of $\Delta_{u}$. This is depictedfbelolawing $P$ to be an invertible operator, it still may not be possible to make $\hat{C}_{u_{r}}$ zero when $\hat{D}_{r}$ is zero. For parametric uncertainty, this occurs when $\hat{D}\left(\Delta_{u}\right)$ loses rank at $\Delta_{u}=0$, but not in a neighborhood of $\Delta_{u}=0$. An interpretation is that there may exist algebraic constraints on the integration states at $\Delta_{u}=0$, but not in a neighborhood of $\Delta_{u}=0$. A simple example is depicted in Figure 4 .

The nominal resistance is zero, which implies that the voltages across the capacitors must be nominally equal, but may be different for all other values of $\delta_{r}$. Clearly the problem arises due to the zero nominal resistance, a poor modeling choice. 


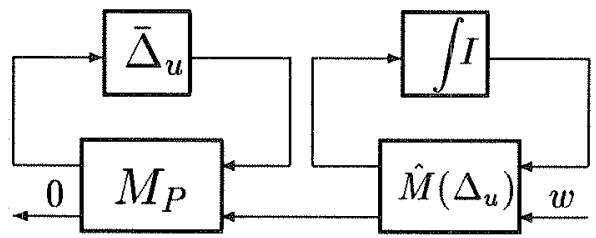

Figure 3: Multiplying by $P\left(\bar{\Delta}_{u}\right)$

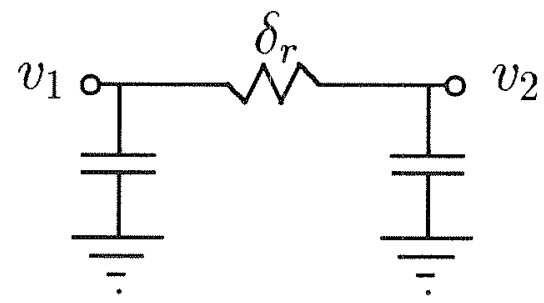

Figure 4: System without Regular Representation

Issues such as whether physically motivated examples exist for which the procedure breaks down, how to deal with full uncertainty blocks when using the augmented reduction procedure, and how to reduce the complexity of the augmented procedure are open research questions.

Non-regular representations which can be reduced to regular representations will be called singular, which generalizes the previous definition of singular.

\section{Input-Output Map Representation}

As discussed in [1], it may be required to represent the behavior of a system $\Sigma_{\Delta}$ in inputoutput form. This may be the case if existing robust control methodologies are to be used for the purpose of designing robust controllers, as these methods require a distinction between system inputs and outputs. The reader is referred to [2] for a characterization of input and output variables. We will restrict ourselves to input-output maps which are nominally proper.

Given a GON representation, we need to know how many variables may be chosen as inputs.

Proposition 4 Given a regular representation $(\Delta, M)$ with $D \in \mathbb{R}^{r \times q}$, any input-output map must have $r$ outputs and $q-r$ inputs.

\section{Proof:}

First, note that the input-output map must be valid for the nominal system. Thus we can restrict ourselves to the case of no uncertainty. Then it is a simple matter to convert the $\mathrm{ON}$ representation to an AR representation, and then invoking Theorem VIII.7 in [2] (which states that an AR representation with $r$ independent equations must have $r$ outputs). 
Let $(\Delta, M)$ be a regular representation of $\Sigma_{\Delta}$. We then have the following:

$$
\begin{aligned}
& p=A \Delta p+B w \\
& 0=C \Delta p+D w
\end{aligned}
$$

Since $D$ is surjective, there exists permutation matrix $\Pi=\left[\Pi_{1} \Pi_{2}\right]$ such that $D \Pi_{1}$ is invertible. Let $w=\Pi\left[\begin{array}{l}y \\ u\end{array}\right]$. Then we have

$$
\begin{aligned}
& p=A \Delta p+B_{1} y+B_{2} u \\
& 0=C \Delta p+D_{1} y+D_{2} u
\end{aligned}
$$

where

$$
B_{1}=B \Pi_{1}, B_{2}=B \Pi_{2}, D_{1}=D \Pi_{1}, D_{2}=D \Pi_{2}
$$

Isolating $y$ and substituting in the $p$ equation yields

$$
\begin{aligned}
& p=\left(A-B_{1} D_{1}^{-1} C\right) \Delta p+\left(B_{2}-B_{1} D_{1}^{-1} D_{2}\right) u \\
& y=-D_{1}^{-1} C \Delta p-D_{1}^{-1} D_{2} u
\end{aligned}
$$

Note that the above is in the form of an LFT relating $y$ and $u$. As of yet, all operations performed have been behavior preserving. If, however, it is required that $u$ vary freely and determine $y$, it must be possible to solve for $p$, ie., for a given $u \in L_{2}^{l o c}$, there must exist a $p \in L_{2}^{l o c}$. This might not be the case for all possible values of $\Delta$ in $\Delta$. We have the following main result:

Theorem 1 Given regular GON representation $(\Delta, M)$ of $\Sigma_{\Delta}=\left(\mathbb{R}^{+}, \mathbb{R}^{q}, \Delta, \mathcal{B}_{\Delta}\right)$, there exists a partition of $\mathbb{R}^{q}=\mathbb{R}^{p} \times \mathbb{R}^{m}$, a permutation matrix $\Pi$, a constant matrix $M_{I / O}$, and a number $\epsilon$ strictly greater than zero such that

$$
\mathcal{B}_{\Delta}=\left\{\Pi\left[\begin{array}{c}
y \\
u
\end{array}\right], y \in L_{2}^{\text {loc }}\left(\mathbb{R}^{p}\right), u \in L_{2}^{\text {loc }}\left(\mathbb{R}^{m}\right) \mid y=\mathcal{S}\left(\Delta, M_{I / O}\right) u\right\}
$$

for all $\Delta \in \mathbf{B}_{\Delta, \epsilon} .\left(\Delta, M_{I / O}, \Pi\right)$ will be referred to as an $\mathrm{I} / \mathrm{O}$ representation of $\Sigma_{\Delta}$.

Proof: Consider the following equations

$$
\begin{aligned}
\dot{x} & =A_{n} x+A_{n u} \Delta_{u} z+B_{n} u \\
z & =A_{u n} x+A_{u} \Delta_{u} z+B_{u} u \\
y & =C_{n} x+C_{u} \Delta_{u} z+D_{I / O} u
\end{aligned}
$$

which correspond to (28) when the matrices are partitioned into their corresponding nominal and uncertain parts, and $p=(\dot{x}, z)$. It is clear that for sufficiently small $\Delta_{u},\left(I-A_{u} \Delta_{u}\right)$ will be invertible. In other words, there exists an $\epsilon>0$ such that $\left(I-A_{u} \Delta_{u}\right)$ is invertible for $\left\|\Delta_{u}\right\|<\epsilon$. We then have the following 


$$
\dot{x}=L x+\Theta x+f
$$

where

$$
\begin{aligned}
L & =A_{n} \\
\Theta & =A_{n u} \Delta_{u}\left(I-A_{u} \Delta_{u}\right)^{-1} A_{u n} \\
f & =\left(A_{n u} \Delta_{u}\left(I-A_{u} \Delta_{u}\right)^{-1} B_{u}+B_{n}\right) u
\end{aligned}
$$

We have the following technical lemma, whose proof may be found at the end of this section, which is central to proving the theorem:

Lemma 2 Given $f \in L_{2}^{l o c}, \Theta: L_{2}^{\text {loc }} \rightarrow L_{2}^{\text {loc }},\|\Theta\| \leq M<\infty, \Theta$ causal and linear, and $L$ a constant square matrix, $\forall$ initial conditions $x(0) \equiv \overline{x_{0}} \exists$ unique $x(t)$ for $t \geq 0$ such that $x(t)$ solves (31) a.e..

Invoking Lemma 2, we conclude that $x$ can be determined from $u$, and that $x \in L_{2}^{\text {Ioc }}$. It then follows from (30) that $z$ can also be solved for, and that $z \in L_{2}^{\text {loc }}$. Finally, since $y$ is a bounded function of $x, z$, and $u, y$ must also be in $L_{2}^{\text {loc }}$.

We then have the following corollary:

Corollary 1 A valid $I / O$ representation matrix $M_{I / O}$ of $\Sigma_{\Delta}$ is

$$
M_{I / O}=\left[\begin{array}{ll}
A_{I / O} & B_{I / O} \\
C_{I / O} & D_{I / O}
\end{array}\right]=\left[\begin{array}{cc}
A-B_{1} D_{1}^{-1} C & B_{2}-B_{1} D_{1}^{-1} D_{2} \\
-D_{1}^{-1} C & -D_{1}^{-1} D_{2}
\end{array}\right]
$$

How can we get a good bound for $\epsilon$ ? One way is to perform a $\mu$ test on the matrix $A_{u}$ with block structure $\Delta_{\mathbf{u}}$. By the definition of $\mu$, see [10], $\epsilon=\frac{1}{\mu_{\Delta_{\mathbf{u}}}\left(A_{u}\right)}$ is the largest value for which $\left(I-A_{u} \Delta_{u}\right)$ is invertible for $\left\|\Delta_{u}\right\|<\epsilon$. We then have the following corollary:

Corollary $2 A$ value of $\epsilon$ consistent with Theorem 1 is $\frac{1}{\mu_{\Delta_{u}}\left(A_{u}\right)}$, where

$$
A_{I / O}=\left[\begin{array}{cc}
A_{n} & A_{u n} \\
A_{n u} & A_{u}
\end{array}\right]
$$

Proof of Lemma 2: Pick an interval $[0, T]$, where $T$ is to be chosen. Define $x_{0}(t) \equiv \overline{x_{0}}$, and

For $n \geq 1$

$$
x_{n+1}(t)=\overline{x_{0}}+\int_{0}^{t}\left(L x_{n}(\tau)+\left(\Theta x_{n}\right)(\tau)+f(\tau)\right) d \tau, n \geq 0,0 \leq t \leq T
$$

$$
\begin{aligned}
\left|x_{n+1}(t)-x_{n}(t)\right| & \leq \int_{0}^{t}\left(\left|L\left(x_{n}(\tau)-x_{n-1}(\tau)\right)\right|+\left|\left(\Theta\left(x_{n}-x_{n-1}\right)\right)(\tau)\right|\right) d \tau \\
& \leq \bar{\sigma}(L) t^{\frac{1}{2}}\left\|x_{n}-x_{n-1}\right\|_{2[0, t]}+t^{\frac{1}{2}}\left\|\Theta\left(x_{n}-x_{n-1}\right)\right\|_{2[0, t]} \\
& \leq(\bar{\sigma}(L)+M) t^{\frac{1}{2}}\left\|x_{n}-x_{n-1}\right\|_{2[0, t]}
\end{aligned}
$$

which implies 


$$
\left\|x_{n+1}-x_{n}\right\|_{2[0, T]} \leq \frac{T}{\sqrt{2}}(\bar{\sigma}(L)+M)\left\|x_{n}-x_{n-1}\right\|_{2[0, T]}
$$

Now choose $T$ to make $\frac{T}{\sqrt{2}}(\bar{\sigma}(L)+M)<1$. Then consider

$$
x(t)=x_{0}(t)+\sum_{n=0}^{\infty}\left(x_{n+1}(t)-x_{n}(t)\right) \in L_{2}^{[0, T]}
$$

Then it follows that

$$
x_{n}(t) \stackrel{L_{2}^{[0, T]}}{\longrightarrow} x(t)
$$

Taking limits on equation (34), and using the continuity of $\Theta$ and integration operators in $L_{2}^{[0, T]}$, yields

$$
x(t)=\bar{x}_{0}+\int_{0}^{t}(L x(\tau)+(\Theta x)(\tau)+f(\tau)) d \tau(\text { a.e. })
$$

which implies that $x$ solves (31) a.e. in $[0, T]$. The argument above can be easily extended to show uniqueness.

It remains to see whether we can extend the solution $x$ to the real line. Clearly, it suffices to show we can do so in the interval $[0,2 T]$. Define

and

$$
x_{0}(t)= \begin{cases}x(t) & , 0 \leq t \leq T \\ 0 & , T<t \leq 2 T\end{cases}
$$

Now, for $t \leq T, x_{n+1}(t)=x(t)$. This uses the fact that $x(t)$ solves (41) in $[0, T]$, and that $\Theta$ is causal. Then we have, for $T \leq t \leq 2 T$ and $n \geq 1$,

$$
\left|x_{n+1}(t)-x_{n}(t)\right| \leq(\bar{\sigma}(L)+M)\left\|x_{n}-x_{n-1}\right\|_{2[T, t]}(t-T)^{\frac{1}{2}}
$$

At this point, the same argument as before applies, and we have a solution $x(t)$ on $[0,2 T]$.

\section{Example}

The following example illustrates some of the various techniques and algorithms presented in the previous sections. It consists of the interconnection of two mechanical systems with uncertain parameters. Define the parameters as

$$
\begin{array}{lll}
k_{1}=1+\delta_{k_{1}}, & b_{1}=1+\delta_{b_{1}}, & m_{1}=1+\delta_{m_{1}} \\
k_{2}=1+\delta_{k_{2}}, & b_{2}=0+\delta_{b_{2}}, & m_{2}=2+\delta_{m_{2}}
\end{array}
$$

The uncertainty can take on various forms. For example, the damping of system 1 could be interpreted as being nominally 1 , with a constant or time varying perturbation to the nominal damping. It could also be interpreted as being nominally 1 with the addition of an operator that acts on the velocity of the mass and converts it to a force.

The equations of motion governing the two systems are

$$
\begin{aligned}
& f_{1}=\left(1+\delta_{m_{1}}\right) \ddot{x_{1}}+\left(1+\delta_{b_{1}}\right) \dot{x_{1}}+\left(1+\delta_{k_{1}}\right) x_{1} \\
& f_{2}=\left(2+\delta_{m_{2}}\right) \ddot{x_{2}}+\delta_{b_{2}} \dot{x_{2}}+\left(1+\delta_{k_{2}}\right) x_{2}
\end{aligned}
$$




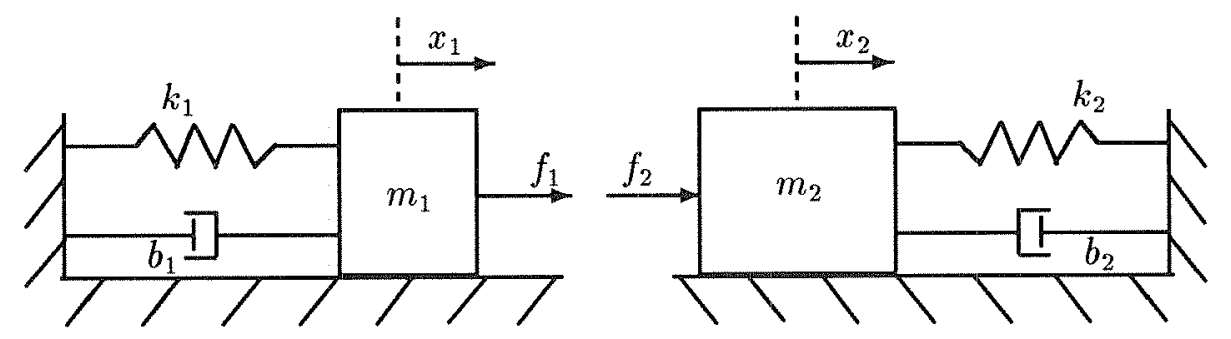

SYSTEM 1 Figure 5: Systems 1 and 2 SYSTEM 2

In order to construct GON representations of the two systems, we need to modify the procedure outlined in the representation section to allow for uncertainty. A GON representation of system 1 can be constructed as follows:

Define

$$
\begin{aligned}
z_{1} & =x_{1} \\
z_{2} & =\dot{x_{1}} \\
z_{3} & =\delta_{k_{1}} z_{1} \\
z_{4} & =\delta_{b_{1}} z_{2} \\
z_{5} & =\left(1+\delta_{m_{1}}\right)^{-1} \delta_{m_{1}}\left(z_{1}+z_{2}+z_{3}+z_{4}-f_{1}\right) \\
& =\delta_{m_{1}}\left(z_{1}+z_{2}+z_{3}+z_{4}-z_{5}-f_{1}\right)
\end{aligned}
$$

then

$$
\dot{z}_{2}=z_{5}-\left(z_{1}+z_{2}+z_{3}+z_{4}-f_{1}\right)
$$

We then have the following equations

$$
\begin{aligned}
& p=A_{1} \Delta_{1} p+B_{1} w_{1} \\
& 0=C_{1} \Delta_{1} p+D_{1} w_{1}
\end{aligned}
$$

where

$$
\begin{aligned}
& z=\left[z_{1}, z_{2}, z_{3}, z_{4}, z_{5}\right]=\Delta_{1} p \\
& A_{1}=\left[\begin{array}{ccccc}
0 & 1 & 0 & 0 & 0 \\
-1 & -1 & -1 & -1 & 1 \\
1 & 0 & 0 & 0 & 0 \\
0 & 1 & 0 & 0 & 0 \\
1 & 1 & 1 & 1 & -1
\end{array}\right], B_{1}=\left[\begin{array}{cc}
0 & 0 \\
0 & 1 \\
0 & 0 \\
0 & 0 \\
0 & -1
\end{array}\right] \\
& C_{1}=\left[\begin{array}{lllll}
1 & 0 & 0 & 0 & 0
\end{array}\right], D_{1}=\left[\begin{array}{ll}
-1 & 0
\end{array}\right] \\
& \Delta_{\mathbf{1}}=\left\{\operatorname{diag}\left[\int I_{2}, \delta_{k_{1}}, \delta_{b_{1}}, \delta_{m_{1}}\right]\right\} \\
& w_{1}=\left[x_{1}, f_{1}\right]
\end{aligned}
$$


which gives us the required regular GON representation of system 1. A similar construction can be performed for system 2 , yielding

$$
\begin{aligned}
& A_{2}=\left[\begin{array}{ccccc}
0 & 1 & 0 & 0 & 0 \\
\frac{-1}{2} & 0 & \frac{-1}{2} & \frac{-1}{2} & 1 \\
1 & 0 & 0 & 0 & 0 \\
0 & 1 & 0 & 0 & 0 \\
\frac{1}{4} & 0 & \frac{1}{4} & \frac{1}{4} & \frac{-1}{2}
\end{array}\right], B_{2}=\left[\begin{array}{cc}
0 & 0 \\
0 & \frac{1}{2} \\
0 & 0 \\
0 & 0 \\
0 & \frac{-1}{4}
\end{array}\right] \\
& C_{2}=\left[\begin{array}{lllll}
1 & 0 & 0 & 0 & 0
\end{array}\right], D_{2}=\left[\begin{array}{ll}
-1 & 0
\end{array}\right] \\
& \Delta_{\mathbf{2}}=\left\{\operatorname{diag}\left[\int I_{2}, \delta_{k_{2}}, \delta_{b_{2}}, \delta_{m_{2}}\right]\right\} \\
& w=\left[x_{2}, f_{2}\right]
\end{aligned}
$$

Let us define the interconnection of the two systems as follows:

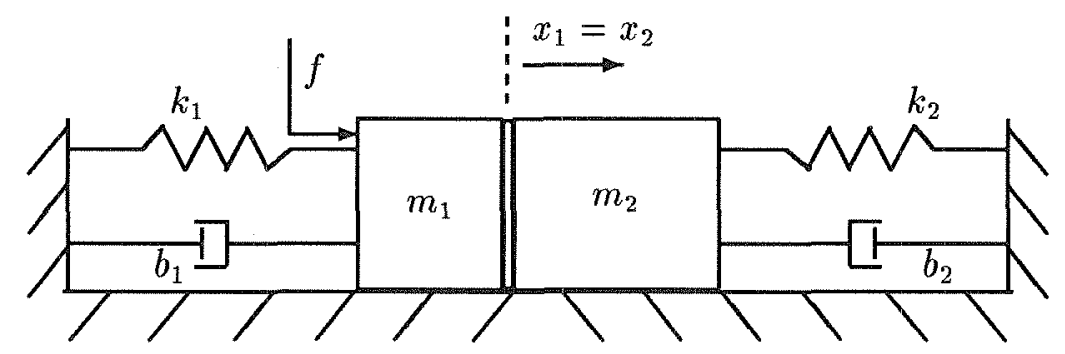

Figure 6: Interconnected system

$$
\begin{aligned}
x_{1} & =x_{2} \\
f_{1}+f_{2} & =f
\end{aligned}
$$

where (49) has a trivial regular GON representation. This corresponds to "bolting" masses 1 and 2 together. Combining systems 1 and 2 with the interconnection constraints results in the following GON representation of the interconnected system:

$$
A=\left[\begin{array}{cccccccccc}
0 & 1 & 0 & 0 & 0 & 0 & 0 & 0 & 0 & 0 \\
-1 & -1 & 0 & 0 & -1 & -1 & 1 & 0 & 0 & 0 \\
0 & 0 & 0 & 1 & 0 & 0 & 0 & 0 & 0 & 0 \\
0 & 0 & \frac{-1}{2} & 0 & 0 & 0 & 0 & \frac{-1}{2} & \frac{-1}{2} & 1 \\
1 & 0 & 0 & 0 & 0 & 0 & 0 & 0 & 0 & 0 \\
0 & 1 & 0 & 0 & 0 & 0 & 0 & 0 & 0 & 0 \\
1 & 1 & 0 & 0 & 1 & 1 & -1 & 0 & 0 & 0 \\
0 & 0 & 1 & 0 & 0 & 0 & 0 & 0 & 0 & 0 \\
0 & 0 & 0 & 1 & 0 & 0 & 0 & 0 & 0 & 0 \\
0 & 0 & \frac{1}{4} & 0 & 0 & 0 & 0 & \frac{1}{4} & \frac{1}{4} & \frac{-1}{2}
\end{array}\right], B=\left[\begin{array}{ccccc}
0 & 0 & 0 & 0 & 0 \\
0 & 0 & 1 & 0 & 0 \\
0 & 0 & 0 & 0 & 0 \\
0 & 0 & 0 & \frac{1}{2} & 0 \\
0 & 0 & 1 & 0 & 0 \\
0 & 0 & 0 & 0 & 0 \\
0 & 0 & -1 & 0 & 0 \\
0 & 0 & 0 & 0 & 0 \\
0 & 0 & 0 & 0 & 0 \\
0 & 0 & 0 & \frac{-1}{4} & 0
\end{array}\right]
$$




$$
\begin{aligned}
C & =\left[\begin{array}{llllllllll}
1 & 0 & 0 & 0 & 0 & 0 & 0 & 0 & 0 & 0 \\
0 & 0 & 1 & 0 & 0 & 0 & 0 & 0 & 0 & 0 \\
0 & 0 & 0 & 0 & 0 & 0 & 0 & 0 & 0 & 0 \\
0 & 0 & 0 & 0 & 0 & 0 & 0 & 0 & 0 & 0
\end{array}\right], D=\left[\begin{array}{ccccc}
-1 & 0 & 0 & 0 & 0 \\
0 & -1 & 0 & 0 & 0 \\
1 & -1 & 0 & 0 & 0 \\
0 & 0 & 1 & 1 & -1
\end{array}\right] \\
\Delta & =\left\{\operatorname{diag}\left[\int I_{4}, \delta_{k_{1}}, \delta_{b_{1}}, \delta_{m_{1}}, \delta_{k_{2}}, \delta_{b_{2}}, \delta_{m_{2}}\right]\right\} \\
w & =\left[x_{1}, x_{2}, f_{1}, f_{2}, f\right]
\end{aligned}
$$

Note that the interconnection is not regular, since $D$ is not surjective. This is expected, since we are forcing the two masses to move together, and thus their positions and velocities are constrained to be equal. Thus we would expect the system to have only two integration states, not four.

After performing the reduction procedure (which terminates after two iterations), we have the following regular GON representation of the interconnected system:

$$
\begin{aligned}
& A=\left[\begin{array}{cccccccc}
\frac{-1}{2} & \frac{-3}{4} & -1 & -1 & 1 & \frac{-1}{2} & \frac{-1}{2} & 1 \\
1 & 0 & 0 & 0 & 0 & 0 & 0 & 0 \\
0 & \frac{1}{2} & 0 & 0 & 0 & 0 & 0 & 0 \\
\frac{1}{2} & 0 & 0 & 0 & 0 & 0 & 0 & 0 \\
\frac{1}{2} & \frac{1}{2} & 1 & 1 & -1 & 0 & 0 & 0 \\
0 & \frac{1}{2} & 0 & 0 & 0 & 0 & 0 & 0 \\
\frac{1}{2} & 0 & 0 & 0 & 0 & 0 & 0 & 0 \\
0 & \frac{1}{8} & 0 & 0 & 0 & \frac{1}{4} & \frac{1}{4} & \frac{-1}{2}
\end{array}\right], B=\left[\begin{array}{ccccc}
0 & 0 & 1 & \frac{1}{2} & 0 \\
0 & 0 & 0 & 0 & 0 \\
0 & 0 & 0 & 0 & 0 \\
0 & 0 & 0 & 0 & 0 \\
0 & 0 & -1 & 0 & 0 \\
0 & 0 & 0 & 0 & 0 \\
0 & 0 & 0 & 0 & 0 \\
0 & 0 & 0 & \frac{-1}{4} & 0
\end{array}\right] \\
& C=\left[\begin{array}{cccccccc}
\frac{-1}{2} & \frac{-1}{4} & -1 & -1 & 1 & \frac{1}{2} & \frac{1}{2} & -1 \\
0 & \frac{1}{2} & 0 & 0 & 0 & 0 & 0 & 0 \\
0 & 0 & 0 & 0 & 0 & 0 & 0 & 0 \\
0 & 0 & 0 & 0 & 0 & 0 & 0 & 0
\end{array}\right], D=\left[\begin{array}{ccccc}
0 & 0 & 1 & \frac{-1}{2} & 0 \\
0 & -1 & 0 & 0 & 0 \\
1 & -1 & 0 & 0 & 0 \\
0 & 0 & 1 & 1 & -1
\end{array}\right] \\
& \Delta=\left\{\operatorname{diag}\left[\int I_{2}, \delta_{k_{1}}, \delta_{b_{1}}, \delta_{m_{1}}, \delta_{k_{2}}, \delta_{b_{2}}, \delta_{m_{2}}\right]\right\} \\
& w=\left[x_{1}, x_{2}, f_{1}, f_{2}, f\right]
\end{aligned}
$$

Using Proposition 4 and Theorem 1, this system must have one input, which is restricted to be one of $f, f_{1}$, or $f_{2}$. Choosing $f$ as an input, Corollary 1 implies

$$
A_{I / O}=\left[\begin{array}{cccccccc}
\frac{-1}{3} & \frac{-2}{3} & \frac{-2}{3} & \frac{-2}{3} & \frac{2}{3} & \frac{-2}{3} & \frac{-2}{3} & 1 \frac{1}{3} \\
1 & 0 & 0 & 0 & 0 & 0 & 0 & 0 \\
0 & \frac{1}{2} & 0 & 0 & 0 & 0 & 0 & 0 \\
\frac{1}{2} & 0 & 0 & 0 & 0 & 0 & 0 & 0 \\
\frac{1}{6} & \frac{1}{3} & \frac{1}{3} & \frac{1}{3} & \frac{-1}{3} & \frac{1}{3} & \frac{1}{3} & \frac{-2}{3} \\
0 & \frac{1}{2} & 0 & 0 & 0 & 0 & 0 & 0 \\
\frac{1}{2} & 0 & 0 & 0 & 0 & 0 & 0 & 0 \\
\frac{1}{12} & \frac{1}{6} & \frac{1}{6} & \frac{1}{6} & \frac{-1}{6} & \frac{1}{6} & \frac{1}{6} & \frac{-1}{3}
\end{array}\right], B_{I / O}=\left[\begin{array}{c}
\frac{2}{3} \\
0 \\
0 \\
0 \\
\frac{-1}{3} \\
0 \\
0 \\
\frac{-1}{6}
\end{array}\right]
$$




$$
\begin{aligned}
C_{I / O} & =\left[\begin{array}{cccccccc}
0 & \frac{1}{2} & 0 & 0 & 0 & 0 & 0 & 0 \\
0 & \frac{1}{2} & 0 & 0 & 0 & 0 & 0 & 0 \\
\frac{1}{3} & \frac{1}{6} & \frac{2}{3} & \frac{2}{3} & \frac{-2}{3} & \frac{-1}{3} & \frac{-1}{3} & \frac{2}{3} \\
\frac{-1}{3} & \frac{-1}{6} & \frac{-2}{3} & \frac{-2}{3} & \frac{2}{3} & \frac{1}{3} & \frac{1}{3} & \frac{-2}{3}
\end{array}\right], D_{I / O}=\left[\begin{array}{c}
0 \\
0 \\
\frac{1}{3} \\
\frac{2}{3}
\end{array}\right] \\
\Delta & =\left\{\operatorname{diag}\left[\int I_{2}, \delta_{k_{1}}, \delta_{b_{1}}, \delta_{m_{1}}, \delta_{k_{2}}, \delta_{b_{2}}, \delta_{m_{2}}\right]\right\} \\
\Pi & =I_{5}
\end{aligned}
$$

where $\Pi$ implies

$$
\begin{aligned}
& y=\left[x_{1}, x_{2}, f_{1}, f_{2}\right] \\
& u=f
\end{aligned}
$$

Several things about the $I / O$ map are worth noting. First of all, the eigenvalues of $A_{I / O_{n}}$ are $-.1667 \pm .7993 i$. These correspond to the natural modes of the unperturbed system, as can be easily verified. Secondly, if we assume that the perturbations are real constants, a Real

$\mu$ test on the uncertainty portion $A_{I / O_{u}}$ of $A_{I / O}$ yields a result of .6667 . This indicates that there exist perturbations of size 1.5 which will cause the $I / O$ representation to be ill-posed. This corresponds to $\delta_{m_{1}}$ and $\delta_{m_{2}}$ being -1.5 , which would make the effective mass of the interconnected system, $m_{1}+m_{2}$, equal to 0 . It is interesting to note that a Real $\mu$ test on system 1 before interconnection would have yielded a value of 1 . This of course corresponds to $\delta_{m_{1}}$ being -1 , and causing the mass to be 0 . The interconnection thus extends the region of validity of the mass uncertainty. This might flag that something non-physical might occur in the interconnected system for a valid value of the uncertainty. For the above example, this reflects the fact that we don't expect mass 1 to be negative, even though the interconnected system is still well posed for a negative value for mass 1 .

\section{Conclusions}

In this paper, an attempt has been made to relate robust control and behavioral paradigms by incorporating structured uncertainty into the description of behavioral systems. This is accomplished by expressing behavioral systems as kernels of operators obtained by an LFT between a constant matrix and an uncertainty structure. An uncertain dynamical system has been defined, and methods of interconnection developed. Finally, a method for obtaining input-output maps from behavioral descriptions of uncertain dynamical systems has been outlined, which makes possible the use of existing robust control methodologies.

\section{Acknowledgements}

The authors would like to acknowledge J. Doyle for motivating this paper, and thank the rest of the Control Group at Caltech for the numerous helpful discussions, especially J. Tierno. We would also like to thank A. Megretski for his input and comments. Finally, we would like to acknowledge AFOSR, NSERC, and NSF as funders of this project. 


\section{References}

[1] D'Andrea R., Paganini F., Doyle J.C., "Uncertain Behavior," Proceedings 1993 CDC, San Antonio, Texas.

[2] Willems J.C., "Paradigms and Puzzles in the Theory of Dynamical Systems," IEEE Transactions on Automatic Control, Vol. 36, pp. 259-294, 1991.

[3] Willems J.C., "Feedback in a Behavioral Setting," Systems, Models and Feedback: Theory and Applications, pp.179-191, 1992.

[4] Weiland S., Theory of Approximation and Disturbance Attenuation for Linear Systems Ph.D. Dissertation Thesis, University of Groningen, 1991.

[5] Packard A., Doyle J.C., Balas G., "Linear, Multivariable Robust Control with a $\mu$-Perspective," Journal of Dynamic Systems Measurement and Control-Transactions of the ASME, Vol. 115, pp. 426-438, 1993.

[6] Kuijper M., First-Order Representations of Linear Systems Ph.D. Dissertation Thesis, University of Brabant, 1992.

[7] Schumacher J.M., "Linear System Representations," Lecture Notes in Control and Information Sciences, Vol 135, pp. 382-408, Springer-Verlag, 1989.

[8] Beck C., Doyle J.C., "Model Reduction of Behavioral Systems" Proceedings 1993 CDC, San Antonio, Texas.

[9] Verghese G.C. et al., "A Generalized State-Space for Singular Systems," IEEE Transactions on Automatic Control, Vol. 26, No. 4, pp. 811-830, 1981.

[10] Packard A., Doyle J.C., "The Complex Structured Singular Value," Automatica, Vol. 29, No. 1, pp. 71-109, 1993. 